\title{
A Wearable Low-cost Muscle Activated Switch for Wireless Control for the Physically Challenged Patients
}

\section{A. Iqbal ( $\square$ abid.iqbal@uetpeshawar.edu.pk)}

University of Engineering and Technology Peshawar University of Engineering \& Technology

\section{Research Article}

Keywords: Myoware sensor, Bluesmirf Bluetooth, Arduino, Paraplegic patient, Grid 3

Posted Date: December 13th, 2021

DOI: https://doi.org/10.21203/rs.3.rs-1146360/v1

License: (c) (i) This work is licensed under a Creative Commons Attribution 4.0 International License.

Read Full License 


\section{Abstract}

\section{Background}

To control the environmental conditions of the living place and to use the electrical appliances and computers etc. are one of the major challenges for the physically disabled persons and elderly people. The surface electro myography (sEMG) is employed to detect the muscle movement and used for games therapy and various others applications. This paper presents a low-cost wearable muscle switch designed to acquire sEMG signal and process it to provides the control, gaming therapy and mode of communication to the physically disabled patients.

Results

This paper proposes a wearable low-cost muscle activated switch to assist the physically challenged members of the society. The designed muscle activated switch consists of Myoware muscle sensors that is used for data acquisition and to detect the muscle activity from the target muscle using Electromyography (EMG) signals. The Arduino board provides the human-computer interface and control to the devices based on the muscle signal. The Bluesmirf bluetooth is used for the wireless connectivity to our designed system that assists the physically disabled persons to use the computer and to control the home appliances via commercially available Grid 3 software and wifi switches. The novelty of this muscle sensor switch is that it can be connected to any bluetooth compatible devices that can be controlled from any targeted muscle. The system was tested with Grid 3 software on laptop for environmental control and text to speech conversion.

\section{Conclusions}

A low cost muscle sensor based Bluetooth switch system for the physically disabled patients has been presented in this paper. The designed switch can be connected to any Bluetooth enabled device as demonstrated in this paper. The physically disable persons can select any module from the Grid 3 software such as the environmental control (to control the environment via electrical appliances), text to speech converter (for Aphasia patients), gaming therapy etc. The designed system can also be utilized to use the Bluetooth enabled electric gadgets such as Mobile Phones, Ipad and android Tablets etc. in accessibility mode.

\section{Background}

In Australia about 4 million people, which is approximately $18 \%$ of the total population have some form of disability and approximately $6.7 \%$ have a severe or profound disability [1]. Human-computer interface is the new growing technology that have gained a lot of significance in order to improve the life quality of persons with disabilities or injuries to perform their duties independently [2]. One of these growing technologies is the field of bionic robot, in which a robot is implanted in humans' body to replace the function of missing human body parts such as the hands or feet etc. These technologies are based on 
bio-signals generated from human body. The most significant bio-signals used to control assistive devices are: Electroencephalogram (EEG) [3], Electrooculography (EOG) [4], Electromyography (EMG) and Electrocardiography (ECG) etc [5-7].

EMG technique is used to record the electrical activity produced by skeletal muscles and to evaluate the specific movements. The EMG signal can be recorded invasively through needle electrodes or noninvasively via placing electrodes on the skin. Generally, the surface electrodes are preferred to avoid the health problems and pain. The Surface electromyography (sEMG) signals are the electrical signals generated by the muscle fibres contractions. The electrodes attached to the skin records these signals in a non-invasive manner [8].

Different research groups have successfully developed multiple sEMG based devices for various applications including prosthetic arm, rehabilitation of limbs amputation, controlling the robots' arm movement $[9,10]$, and muscles based recreational video games [11]. Biagetti et al. designed a low cost wireless system for SEMG acquisition and accelerometer signals for healthcare and fitness applications [12]. Accidents like spinal cord injury (SCl) is often associated with paralysis that can results in the disability of the subject. Also, in other health conditions such as stroke, multiple sclerosis and Guillain-Barré syndrome can cause the muscles weakness or immobility in some body parts.

Consequently, a rehabilitation process and other assistive electronic devices can be beneficial for the subject to performs everyday life functions autonomously [13]. Widhiada et al. successfully demonstrated the myoware sensor for the hybrid control of bionic leg [14]

MyoWare muscle sensors are commercially available and used by numerous research groups. Toro et al. successfully demonstrated that myoware sensor can be used for detect muscle fatigue detection [15]. Khan et al. demonstrated an EMG based real time video game control using myoware sensor for the rehabilitation process of upper limbs muscles [16]. The wheelchair control for paraplegic patients is demonstrated by Dandamudi et al [17]. The finger and hand movement detection is carried out by using Myoware muscle sensors $[18,19]$.

In this paper the muscle-based switch is presented that is made up of Myoware muscle sensor, audrino board, power supply and BlueSmirf Bluetooth module that will assist the physically disabled persons to use the computer and social media apps, to control the home appliances via commercially available Grid 3 software and wifi switches.

\section{Results}

The muscle activated switch connected with GRID 3 operated in switch mode is shown in Fig 1. The grid is selected from main menu according to the user priority to communicate or control the environmental conditions. It can be observed that upon the muscle contraction, the signal is sent to the GRID 3 via bluetooth from the muscle sensor placed on the target muscle of user (patient). The Grid 3 scan vertically upon the first signal sent from the muscle contraction while the horizontal scan on second muscle 
contraction as shown in Figure 1 (b). Upon the third contraction signal from the muscle, the option is selected.

Figure 2 shows the environmental grid selection from the main menu. The Fan, Light and television etc. Grid is selected from the environmental grid in switch mode. The user can select any grid via a muscle switch with limited effort or movement.

Figure 3 shows the symbol talker grid selection from the main menu. This module assists the physically disable patient with speaking difficulty to use muscle switch to select the words from grid for communication.

\section{Discussion}

The design wearable muscle sensor switch is connected successfully to the laptop via Bluetooth as discussed in the result section. The design switch is low cost and can be connected to any Bluetooth enable devices. The design switch can be used with Grid 3 software (installed on any laptop, android or Ipad) for multiple purpose. The different modes can be utilized for the paraplegic patients with limited movement for the games therapy, word to speech conversion (for Aphasia patients), to use social media and cell phones and to control the environmental conditions via wireless switches connected to electrical appliances. Also, the alarm mode in Grid 3 can be used for any emergency to inform the care takers or medical staff in case of any emergency. The novelty of this designed switch is that it can be controlled from any targeted muscle that seems very beneficial for the paraplegic patients and elderly persons, and it can be connected to the other devices via bluetooth module. The lipo battery setup provides the extra advantage of mobility and wireless connectivity to the potential users. The designed switch can also be used with any mobile phones in accessibility mode.

\section{Conclusion}

Science is exploring the power of the latest technological advancements to develop solutions for the communication and controlling the environment issues for physical disabled patients. In this paper, an Electromyography (EMG) based muscle activated switch is presented for wireless control for physically disabled persons to control their environments and to use the gadgets. A standard MyoWare muscle sensor connected with Arduino board that collect and process the data from the target muscle and send the control signals to the Smatgrid via bluesmirf bluetooth device. The physically disabled persons can use the electronic gadgets like Ipad, Laptop, mobile phone etc. via our designed muscle switch to communicate and control the environments via wireless switches. The design muscle sensors will also assist in the gaming therapy for the rehabilitation of the physically challenged patients.

\section{Methods And Materials}


The block diagram of our proposed system is shown in Figure 4. The Myoware muscle sensor is used for data acquisition from muscle sensor according to the patient's physical condition and abilities. This data is fed to the Arduino board which process the muscle sensor data and give the signal to the Bluetooth mate. The Bluetooth mate is used for the Bluetooth communication between the Arduino board which acts as a brain of our system and the laptop, to communicate with Grid 3 software. The Grid 3 software is designed to empowers the people with physical disabilities to communicate, control their environment and access their computer. In our proposed systems the physically challenged patients can control the room environment via wifi switches that can be controlled through the Grid 3 software. Also, the patient can use the social media apps and can-do other activities such as card design etc. The symbol communication feature of Grid 3 can also be utilized that will assists the patients with speaking disability.

Our proposed design of muscle activated switch is shown in Figure 5. The myoware muscle sensor is used from advanced technologies for measuring the muscle activation via electric potential that is also known as electromyography (EMG). The Myoware board gives the output signal in the range of 0-Vs Volts proportional to muscle activity in the selected muscle. It amplifies the voltage difference between the two electrodes on the target muscle, filters and transforms it into the positive voltage domain using a full wave rectifier, and lastly smooth the rectified signal. The EMG/ECG Foam Solid Gel Electrodes are used to stick the electrodes with the patient arm or selected muscle. The 3 electrodes are connected to Myoware Muscle Sensor to measure voltages from muscles when they stretch and shrink, as shown in Fig. 2. Note that these voltages represent the EMG signal strength.

The Arduino Pro Mini microcontroller board based on the ATmega328 as shown in Figure 6, is used as the brain of our proposed system. The Arduino pro mini is employed in our design because of its smaller size of $0.7 "$ " $1.3^{\prime \prime}$ and low power requirements compared to arduino Uno. The Arduino Pro Mini microcontroller is the brain of the muscle-based switch that monitors the muscle sensor and send the controls signals to computer via a Bluetooth module.

The Bluetooth mate from Sparksfun as shown in Figure 7, is used in Human Interface Devices (HID) Mouse configuration for Bluetooth communication between the muscle sensor and other the laptop. The Bluetooth mate handles all the Bluetooth protocols of the Muscle based switch like pairing with and transmitting commands to a Bluetooth capable device e.g., Laptop or tablet etc.

The SparkFun Charger/Booster as shown in figure 8, is employed for powering the whole circuit through Lithium Polymer (Lipo) battery at $5 \mathrm{~V}$. This LiPo charger is equipped with a simple booster circuit that step-up the Lipo battery 3.7 voltage to $5 \mathrm{~V}$ for powering devices and capable of providing a current up to $1 \mathrm{~A}$. The charger/ booster is used to provide the mobility to our design switch.

Grid 3 is a software from the Smartbox Assistive Technology is a that enables the users with physical disabilities to communicate, control their environment and control their computers, laptop or Ipad. The Grid 3 can be used with various alternative access such as eye gaze, switch technology and pointing devices. The designed muscle sensor switch will be used in switch mode with the Grid 3. 


\section{System Architecture And Working}

The complete circuit diagram of Muscle based switch is shown in Figure 9. The signal pin of muscle sensor is connected on Pin AO of Arduino mini pro. The muscle sensor is powered from audrino board via connecting the positive pin to Vcc pin and negative to ground pin respectively. After loading the program sketch in Arduino board, the Bluetooth mate pins are connected to Arduino as shown in Fig 9. The Arduino board is powered via a lipo battery and sparkfun charger/booster to provide immobility to the designed switch. The lipo battery can be recharged via connecting to through usb cable to 5 -volts charger.

The intended muscle area for muscle sensor placement first is thoroughly cleaned, the electrodes are then snapped on the sensor's sensor is placed on the desired target muscle in such a way that one of the connected electrodes is in the middle of the muscle body while the other electrode should line up in the direction of the muscle length. Finally, the reference electrode is placed on a bony or nonadjacent muscular part of near the targeted muscle. The muscle sensor is then connected to the Arduino board and whole circuit.

Upon the powering of muscle switch-based mouse, the red Light emitting diode (LED) on the BluSMiRF module will blink on and off to indicate it is waiting to be paired to a Bluetooth device. The module can be connected to PC, Laptop or tablets etc via pairing it. When the module is connected and paired, the red LED turns of and the green LED turns on continuously while the device remains connected. The green LED can be connected to pin 13 of Arduino board to indicate the muscle flexion. When the user flexes their muscle and the Muscle Sensor's output voltage increases above the threshold value, the Arduino will turn the Green LED on pin 13 on. In other case, it will be set off.

The Grid 3 is used in switch mode where mouse click is used as switch. The switch setting can be selected from the Menu bar, then Settings, Access and Switches and then in switching scanning mode can be selected as shown in Fig 10. Then Switch scanning can be selected whereas the mouse is used as the third-party device single switch.

\section{Abbreviations}

sEMG Surface electro myography

EMG Electromyography

EEG Electroencephalogram

EOG Electrooculography

ECG Electrocardiography

SCI Spinal cord injury 
LiPo Lithium Polymer

HID Human Interface Devices

LED Light emitting diode

\section{Declarations}

Author's contributions The author read and approved the final manuscript.

Acknowledgements Not applicable.

Competing interests The authors declare that they have no competing interests.

Availability of data and materials The datasets used and analysed during the current study are available from the corresponding author on reasonable request.

Consent for publication Not applicable

Ethics approval and consent to participate Not applicable.

Funding Not Applicable.

\section{References}

[1] C. Bigby, C. Tilbury, and M. Hughes, "Social work research in the field of disability in Australia: A scoping review," Australian Social Work, vol. 71, no. 1, pp. 18-31, 2018.

[2] M. A. Oskoei, and H. Hu, "Myoelectric control systems-A survey," Biomedical signal processing and control, vol. 2, no. 4, pp. 275-294, 2007.

[3] K. K. Ang, and C. Guan, "EEG-based strategies to detect motor imagery for control and rehabilitation," IEEE Transactions on Neural Systems and Rehabilitation Engineering, vol. 25, no. 4, pp. 392-401, 2016.

[4] R. Barea, L. Boquete, M. Mazo, and E. López, “Wheelchair guidance strategies using EOG,” Journal of intelligent and robotic systems, vol. 34, no. 3, pp. 279-299, 2002.

[5] H. He, and K. Kiguchi, "A study on EMG-based control of exoskeleton robots for human lower-limb motion assist." pp. 292-295.

[6] N. T. Schutta, "The Impact of Technology on Special Education Students," 1998.

[7] H. Sun, X. Zhang, Y. Zhao, Y. Zhang, X. Zhong, and Z. Fan, "A novel feature optimization for wearable human-computer interfaces using surface electromyography sensors," Sensors, vol. 18, no. 3, 
pp. 869, 2018.

[8] R. Merletti, and P. J. Parker, Electromyography: physiology, engineering, and non-invasive applications. John Wiley \& Sons, 2004.

[9] L. Van Dijk, C. K. Van Der Sluis, H. W. Van Dijk, and R. M. Bongers, "Learning an EMG controlled game: Task-specific adaptations and transfer," PloS one, vol. 11, no. 8, 2016.

[10] E. J. Rechy-Ramirez, A. Marin-Hernandez, and H. V. Rios-Figueroa, "A human-computer interface for wrist rehabilitation: a pilot study using commercial sensors to detect wrist movements," The Visual Computer, vol. 35, no. 1, pp. 41-55, 2019.

[11] X. Zhang, X. Chen, W.-h. Wang, J.-h. Yang, V. Lantz, and K.-q. Wang, "Hand gesture recognition and virtual game control based on 3D accelerometer and EMG sensors." pp. 401-406.

[12] G. Biagetti, P. Crippa, L. Falaschetti, S. Orcioni, and C. Turchetti, "Human activity monitoring system based on wearable sEMG and accelerometer wireless sensor nodes," Biomedical engineering online, vol. 17, no. 1, pp. 1-18, 2018.

[13] C. Wiesener, T. Seel, J. Axelgaard, R. Horton, A. Niedeggen, and T. Schauer, "An Inertial Sensorbased Trigger Algorithm for Functional Electrical Stimulation-Assisted Swimming in Paraplegics," IFACPapersOnLine, vol. 51, no. 34, pp. 278-283, 2019.

[14] W. Widhiada, M. Parameswara, I. N. Santhiarsa, I. Budiarsa, I. Karohika, and I. Suryawan, "HYBRID CONTROL SYSTEM IN BIONIC LEG USING MYOWARE SENSOR," Journal of Southwest Jiaotong University, vol. 56, no. 4, 2021.

[15] S. F. d. Toro, S. Santos-Cuadros, E. Olmeda, C. Álvarez-Caldas, V. Díaz, and J. L. San Román, "Is the Use of a Low-Cost sEMG Sensor Valid to Measure Muscle Fatigue?," Sensors, vol. 19, no. 14, pp. 3204, 2019.

[16] R. U. Khan, M. W. Tahir, and M. I. Tiwana, "Rehabilitation Process of Upper Limbs Muscles through EMG Based Video Game." pp. 1-5.

[17] A. G. B. Dandamudi, D. N. Rao, V. P. Aravilli, and R. Sunitha, "Single Channel Electromyography Controlled Wheelchair Implemented in Virtual Instrumentation." pp. 1040-1045.

[18] A. Junlasat, T. Kamolklang, P. Uthansakul, and M. Uthansakul, "Finger Movement Detection Based on Multiple EMG Positions." pp. 1-4.

[19] Y. Omama, C. Haddad, M. Machaalany, A. Hamoudi, M. Hajj-Hassan, M. Abou Ali, and L. Hamawy, "Surface EMG Classification of Basic Hand Movement." pp. 1-4. 

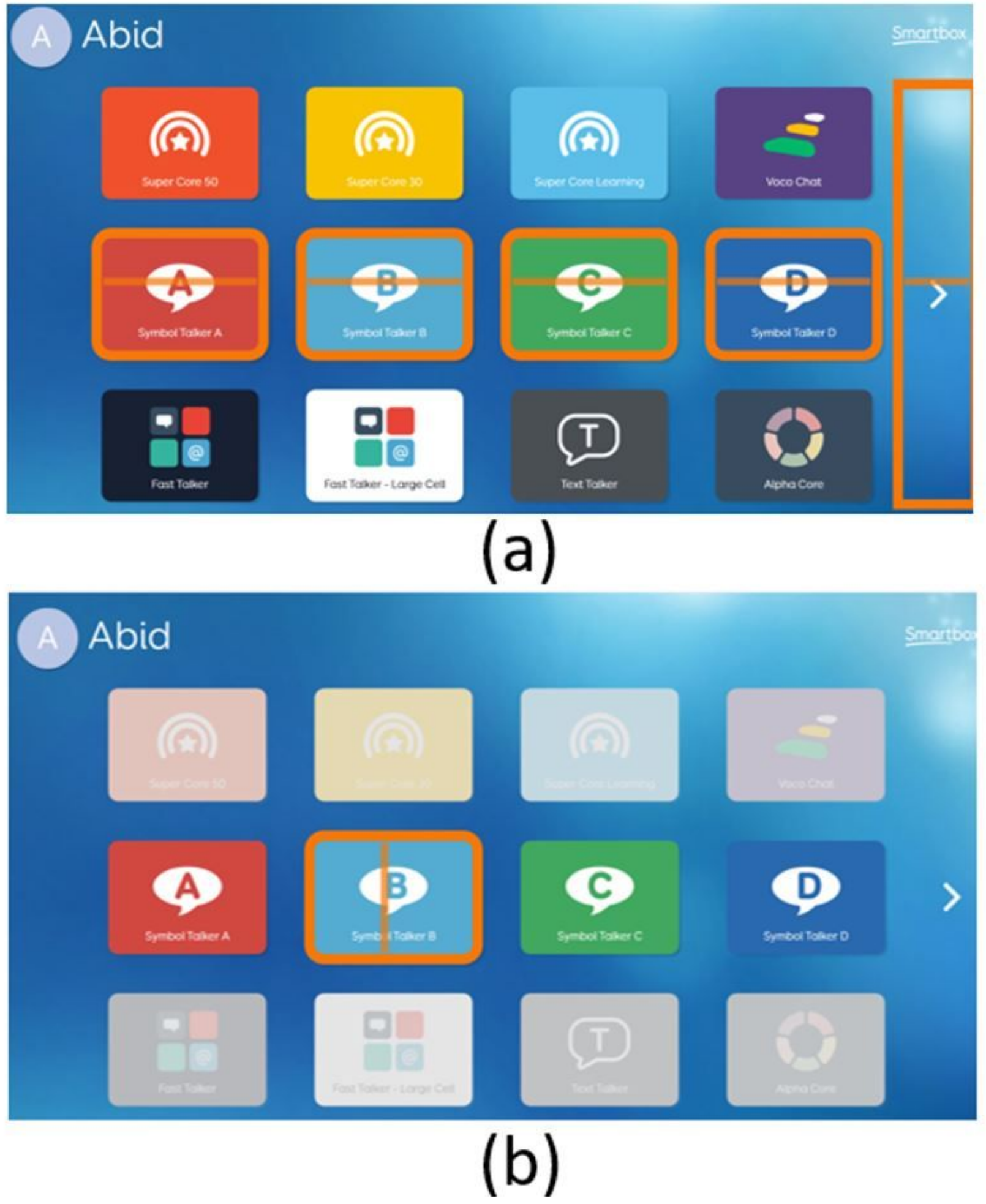

Figure 1

(a) Vertical scan for Grid selection in switch mode (b) Horizontal scan for Grid selection in switch mode 


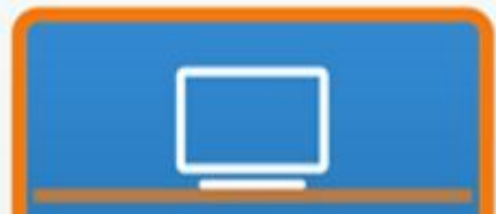

Television

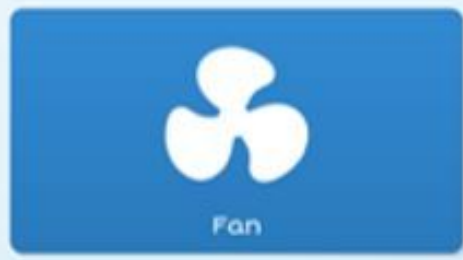

\section{(a)}
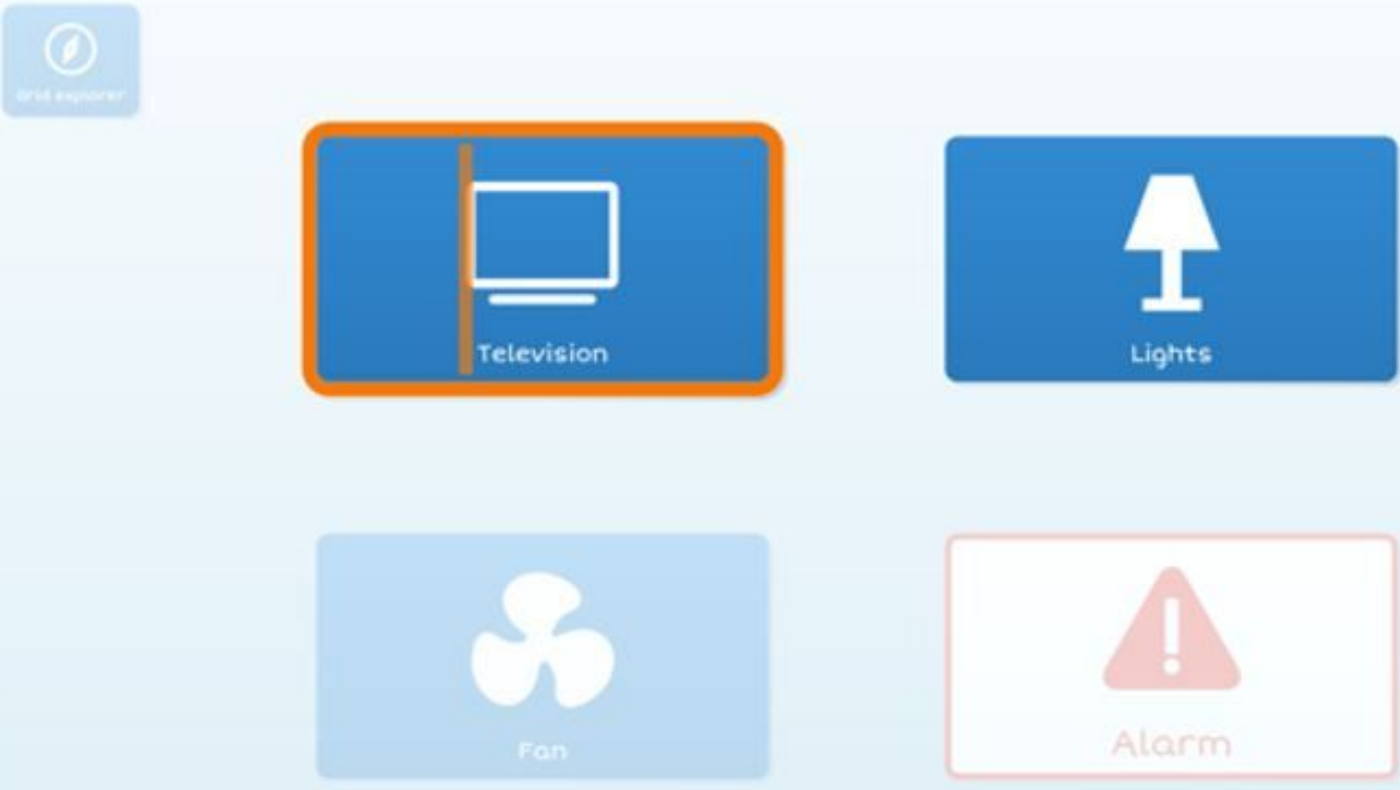

\section{(b)}

Figure 2

(a) Vertical scan in GRID 3 for appliances control selection (b) Horizontal scan in GRID 3 for appliances control selection 

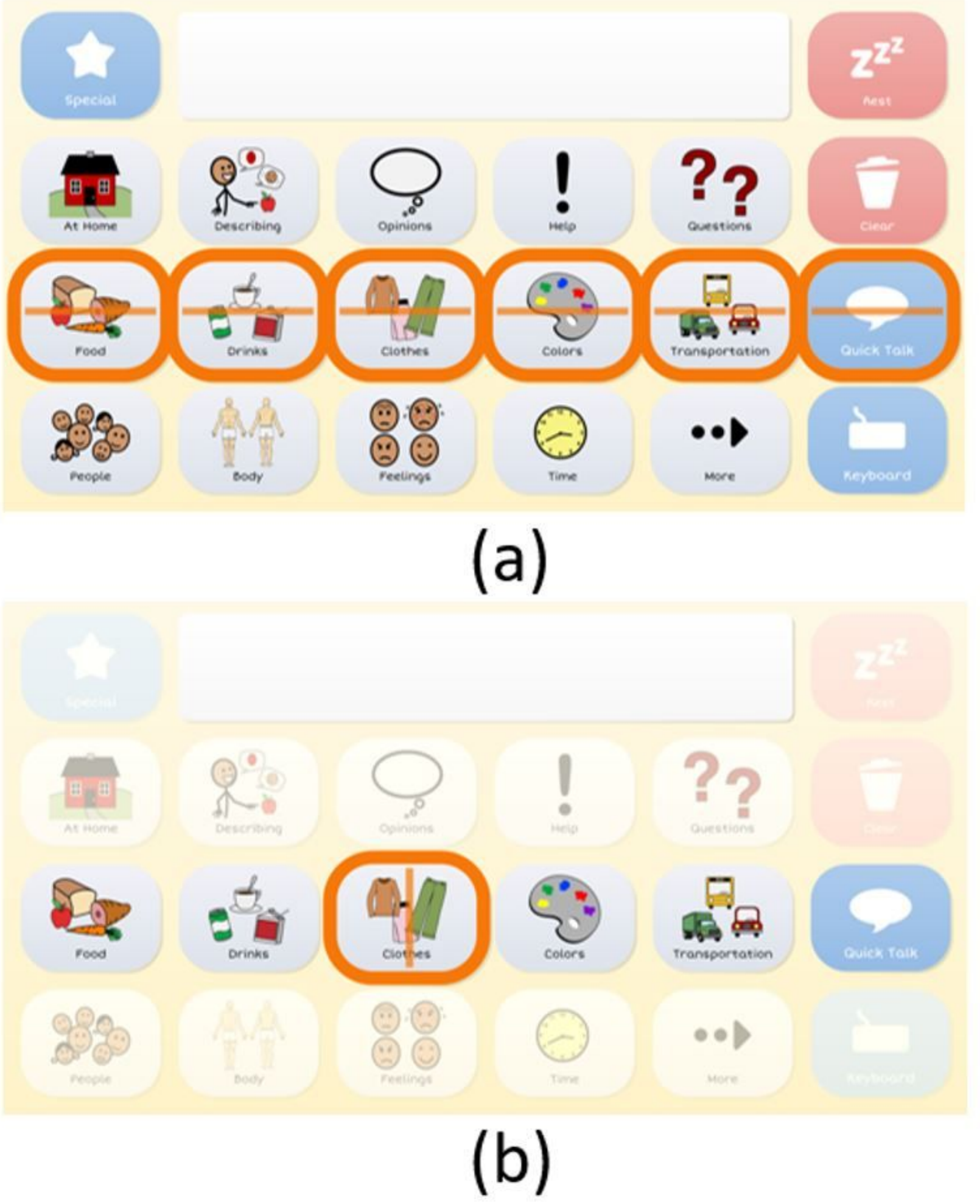

- 1

\section{Figure 3}

(a) Vertical scan in GRID 3 for symbol talker selection (b) Horizontal scan in GRID 3 for symbol talker selection 


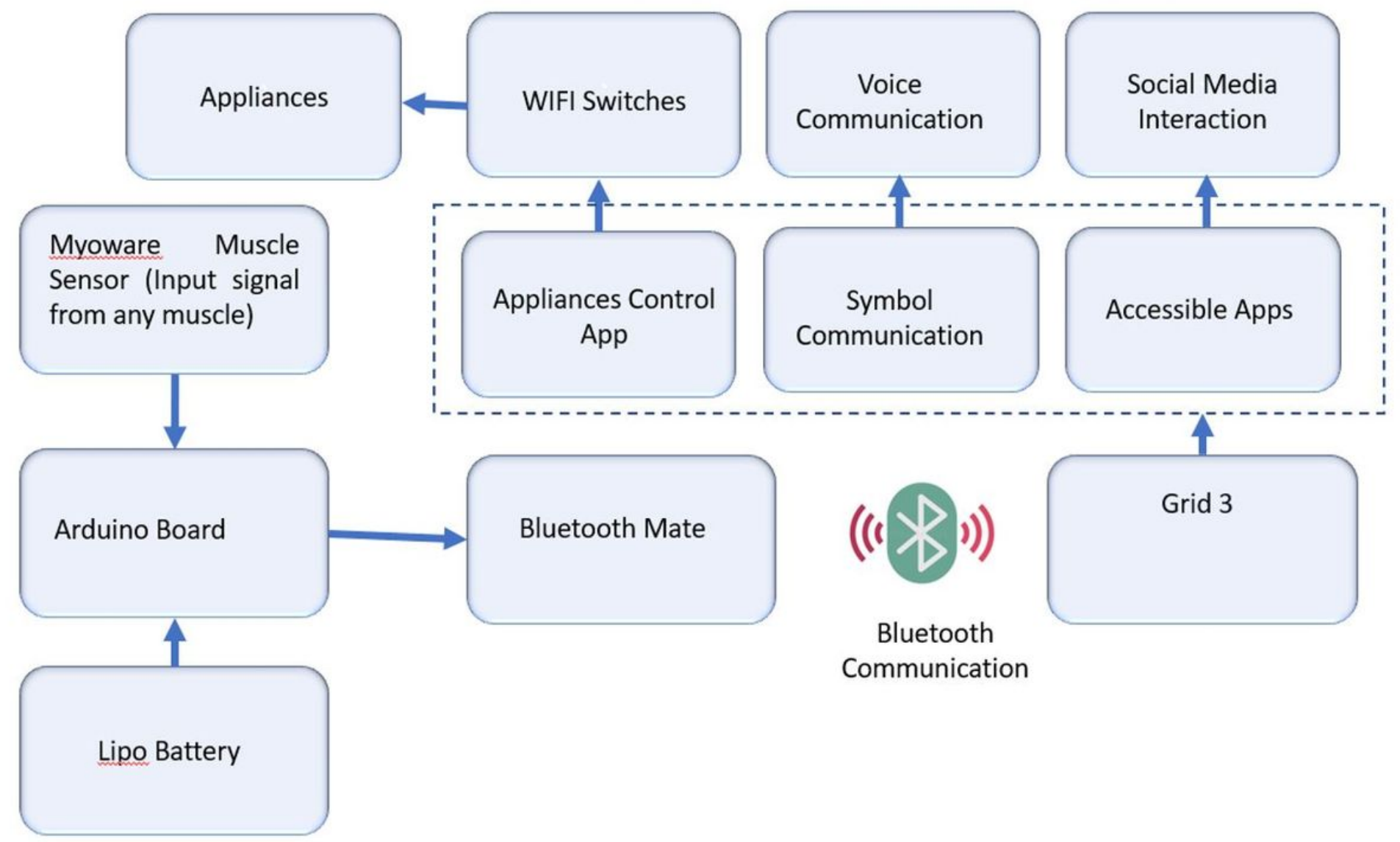

Figure 4

Block Diagram of our proposed model 


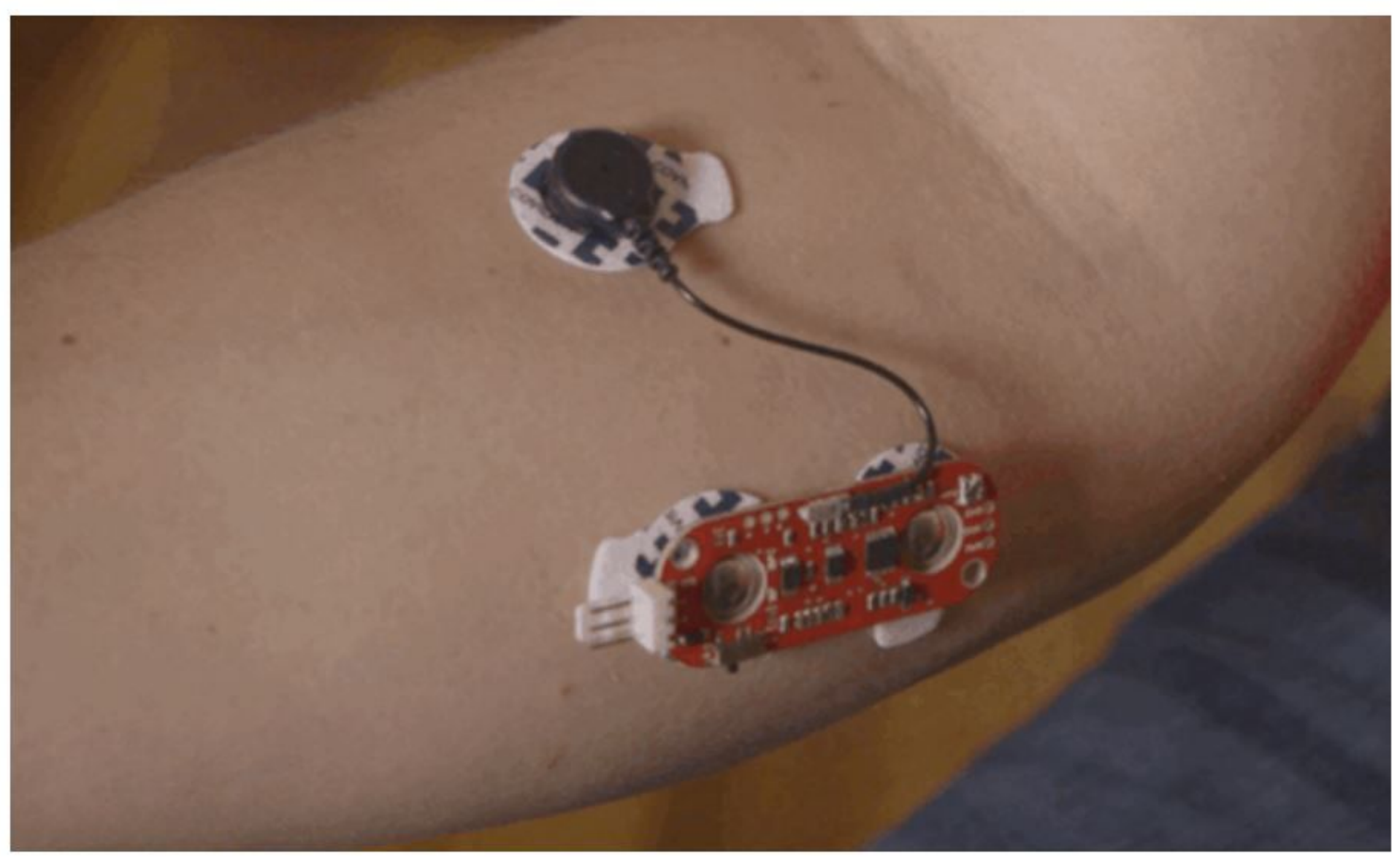

Figure 5

Placing of Myoware muscle sensor on forearms 


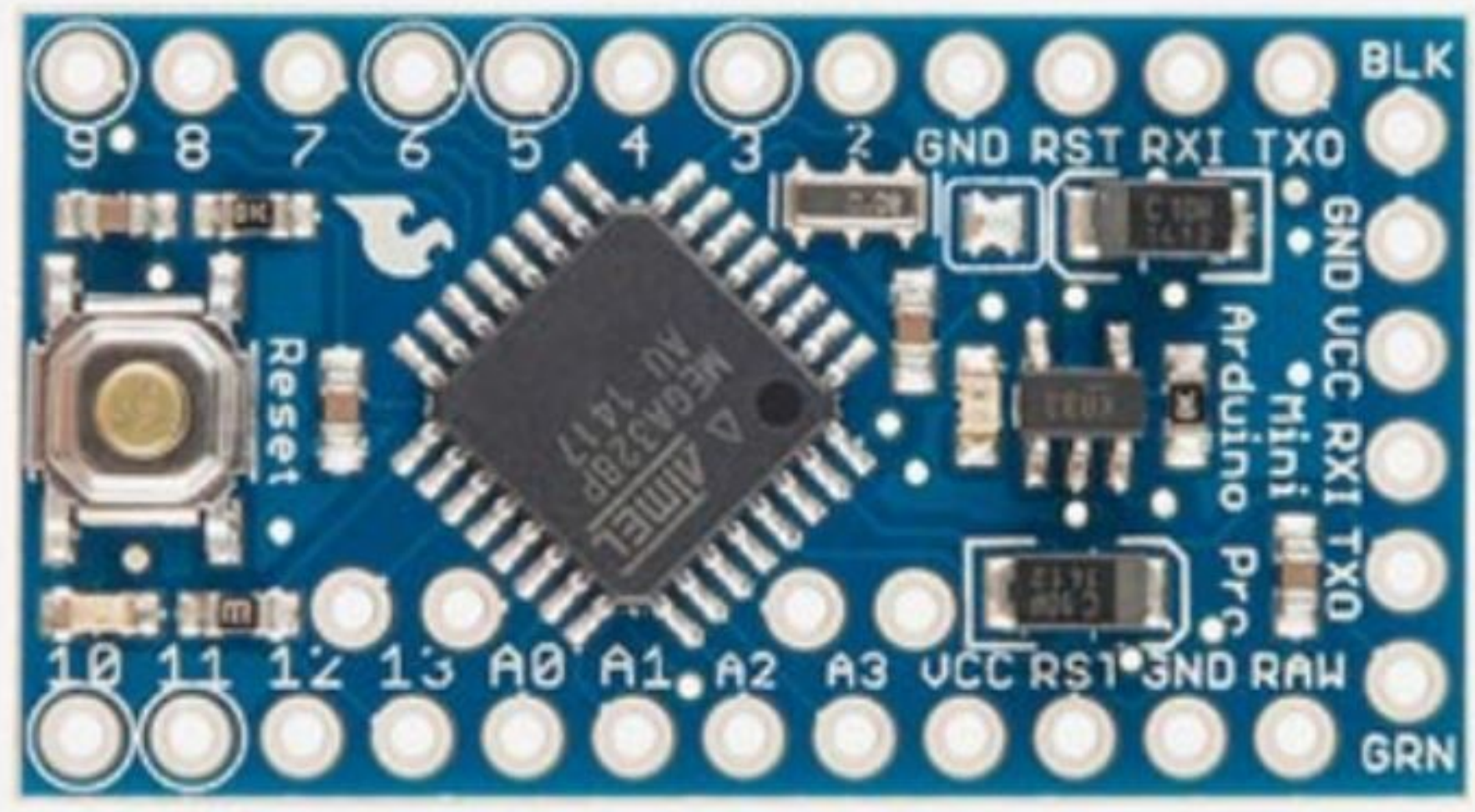

Figure 6

Arduino Mini Pro 


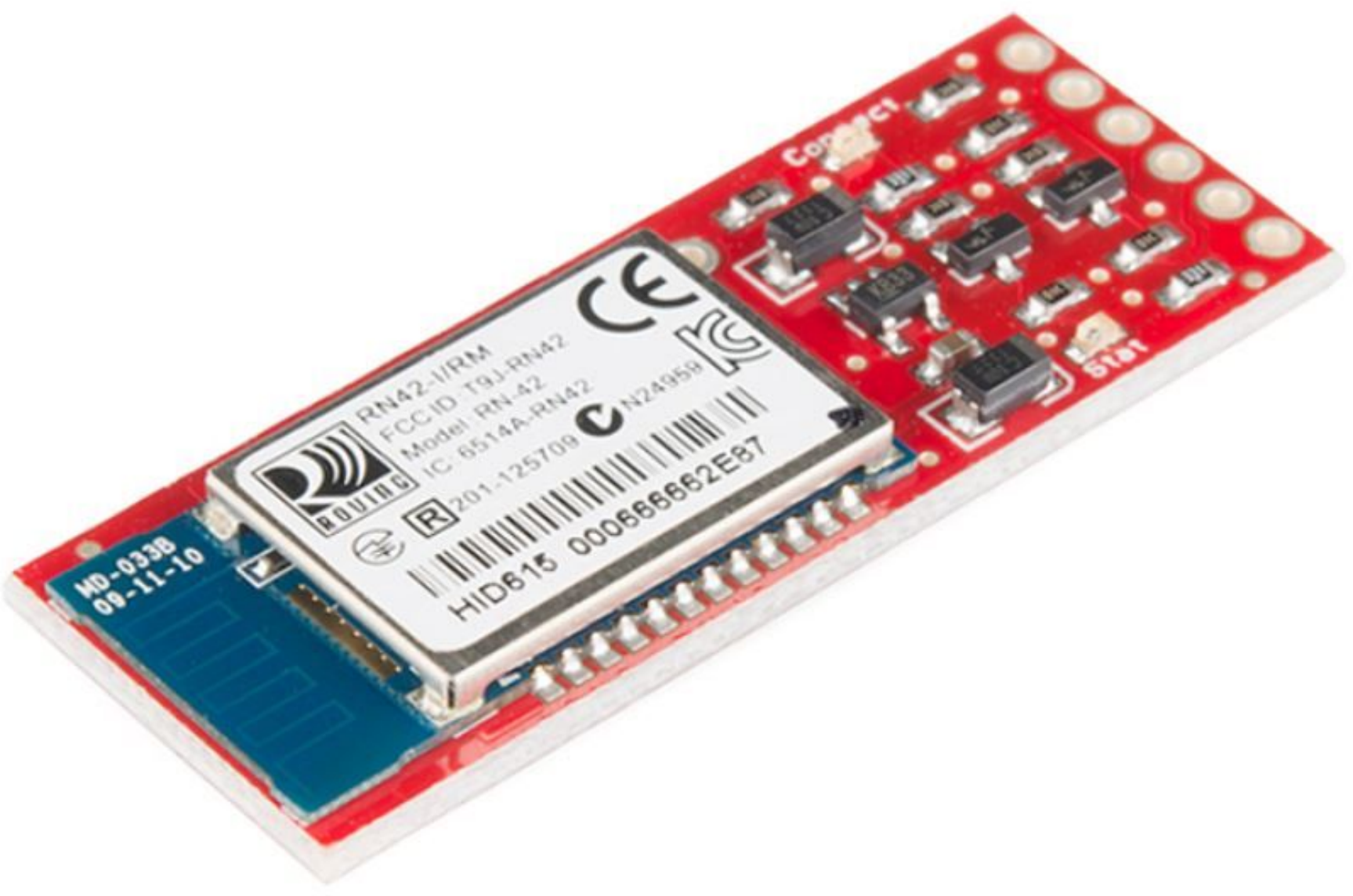

Figure 7

The bluetooth mate from Sparksfun 


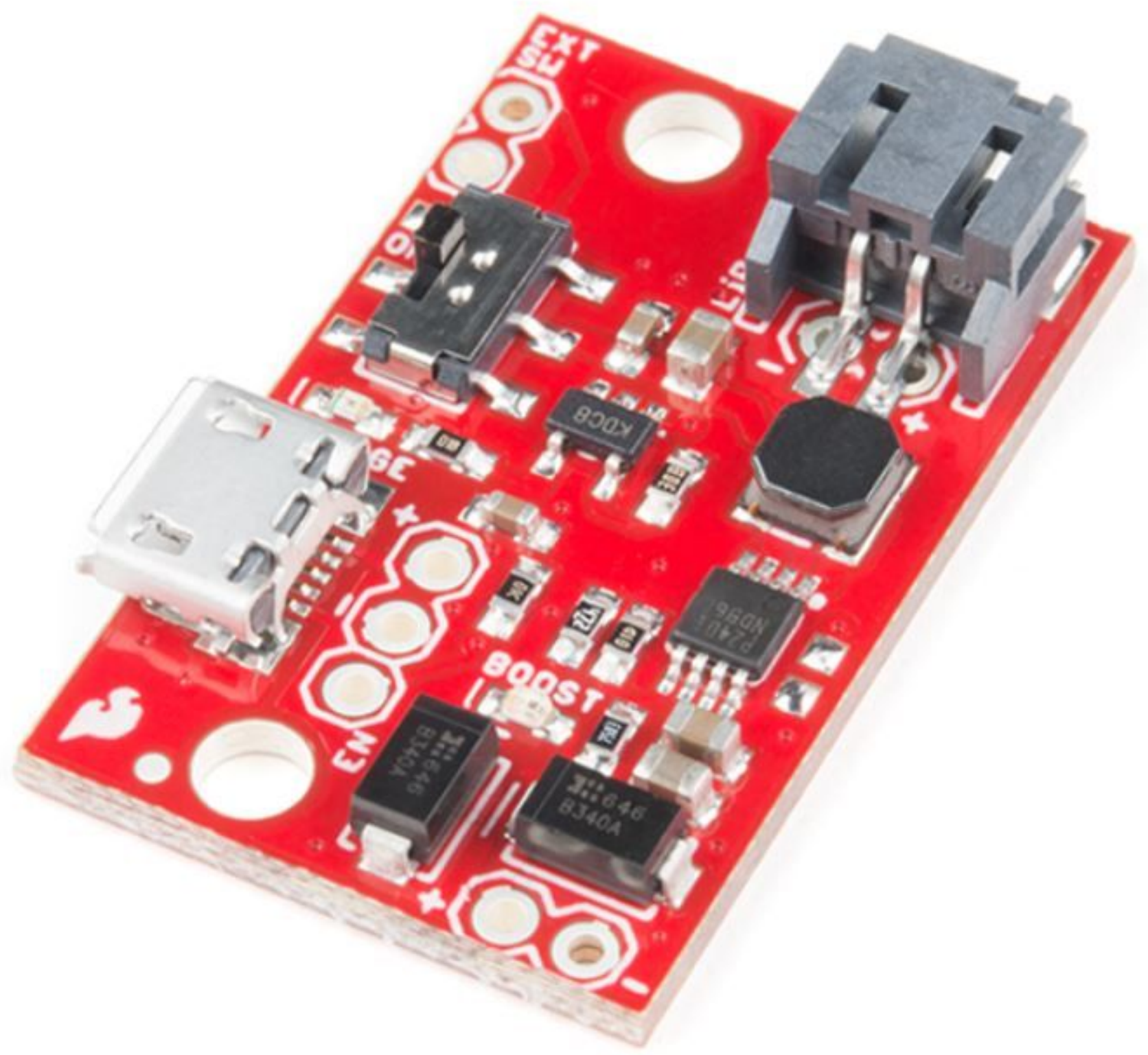

Figure 8

The charge/booster from Sparksfun 


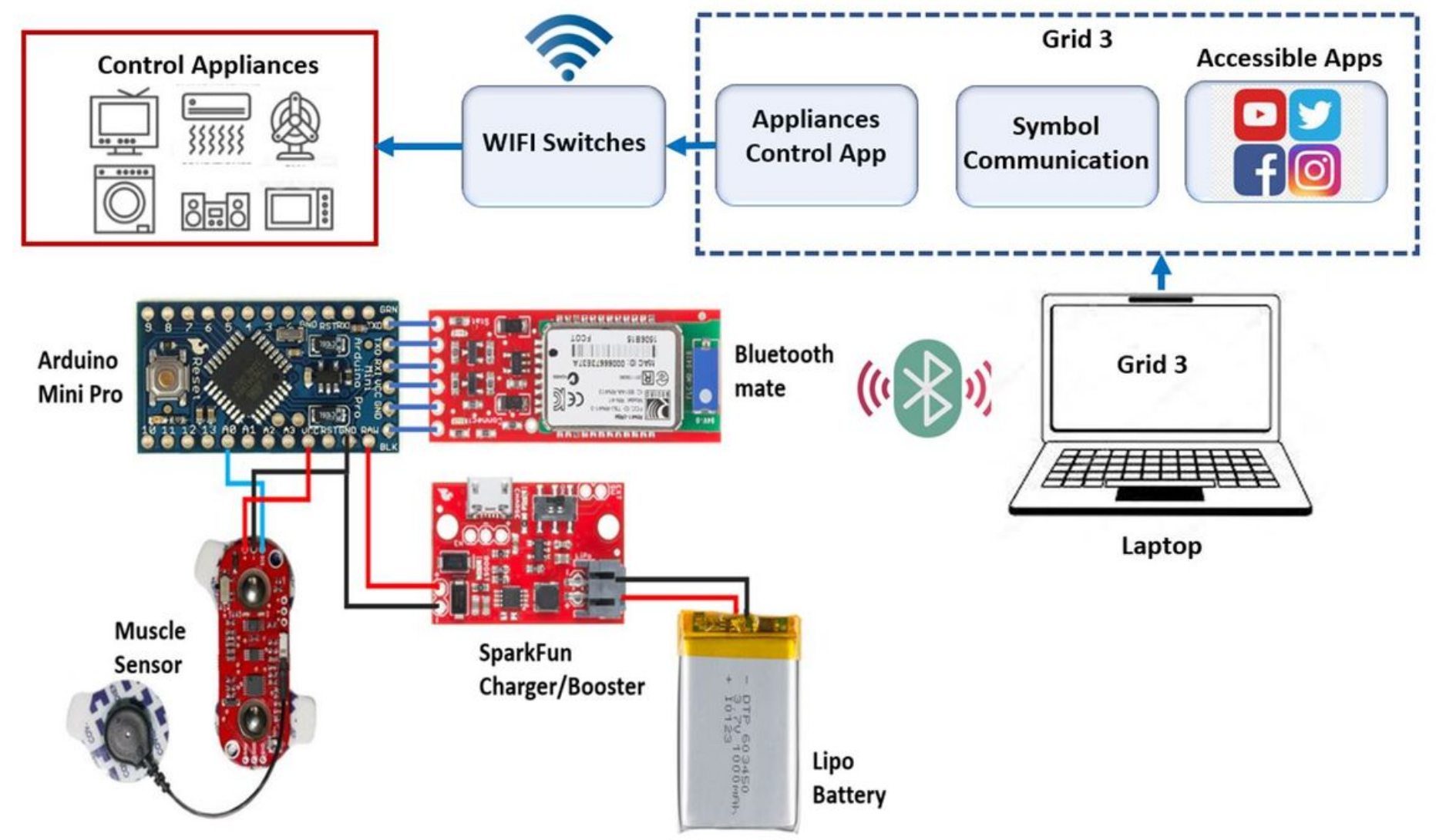

Figure 9

Circuit diagram of the Muscled based switch 


\section{Switches}

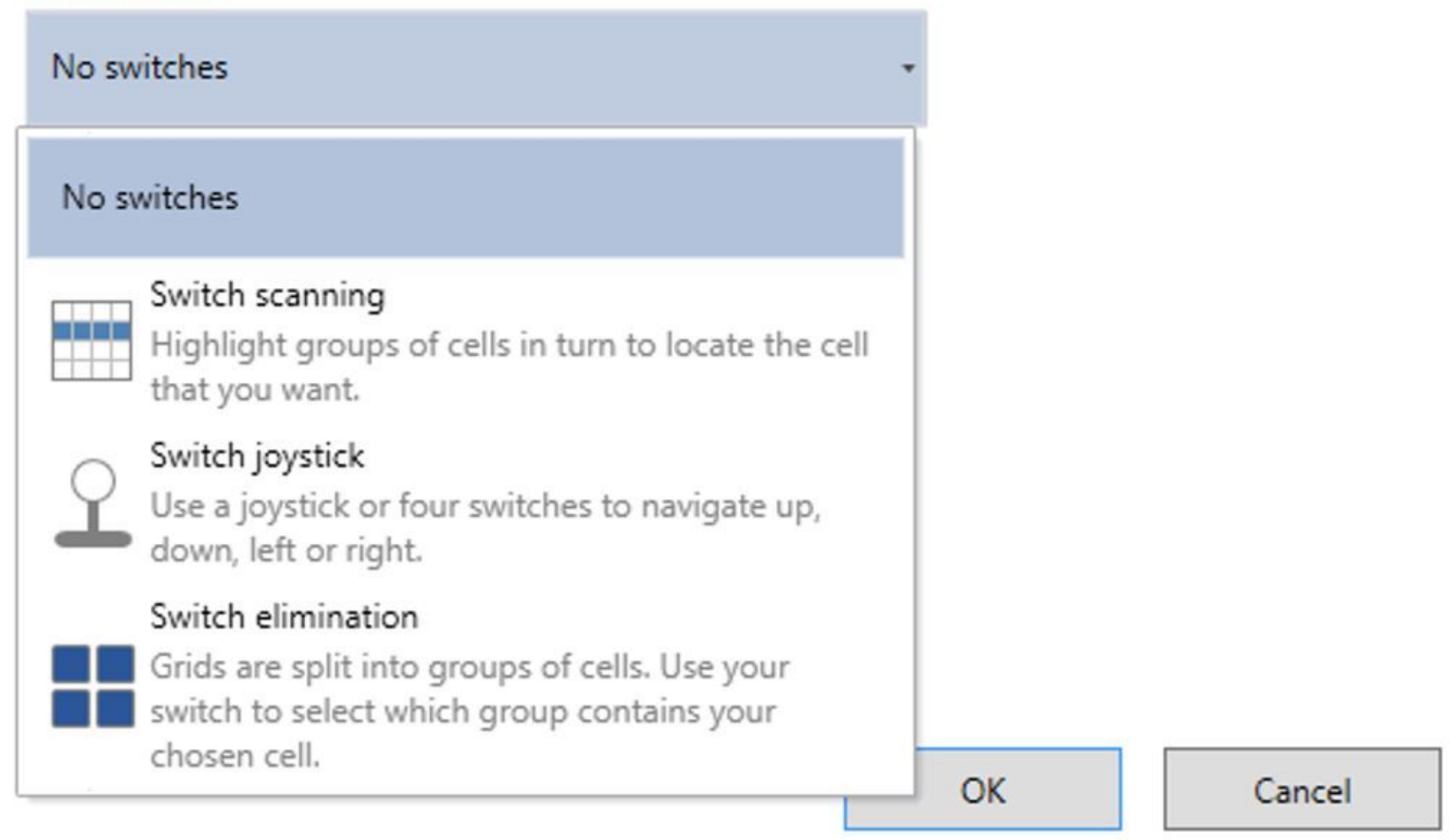

Figure 10

Switch setting in GRID 3 